\title{
Local Gyrokinetic Vlasov Simulations with Realistic Tokamak MHD Equilibria
}

\author{
Motoki NAKATA, Akinobu MATSUYAMA, Nobuyuki AIBA, Shinya MAEYAMA, \\ Masanori NUNAMI ${ }^{1)}$ and Tomo-Hiko WATANABE ${ }^{2)}$ \\ Japan Atomic Energy Agency, Rokkasho, Aomori 039-3212, Japan \\ ${ }^{1)}$ National Institute for Fusion Science, Toki 509-5292, Japan \\ 2) Department of Physics, Nagoya University, Nagoya 464-8602, Japan
}

(Received 15 January 2014 / Accepted 21 February 2014)

\begin{abstract}
A local gyrokinetic Vlasov simulation code GKV is extended to incorporate realistic tokamak equilibria including up-down asymmetry, which are produced by a free-boundary 2D Grad-Shafranov equation solver MEUDAS. By using a newly developed interface code IGS, two dimensional rectangular equilibrium data from MEUDAS is converted to straight-field-line flux coordinates such as Hamada, Boozer, and axisymmetric coordinates, which are useful for gyrokinetic micro-instability and turbulent transport analyses. The developed codes have been verified by a cross-code benchmark test using Cyclone-base-case like MHD equilibrium, where good agreement in the dispersion relation of ion temperature gradient (ITG) driven mode has been confirmed. The extended GKV is applied to two types of shaped plasmas expected in JT-60SA tokamak devices, i.e., ITER-like and highly-shaped plasmas, and ITG-mode stability and residual zonal-flow level are investigated. Through the detailed comparisons, more favorable stability properties against the ITG mode are revealed for the highly-shaped case, where the lower ITG-mode growth rate and higher residual zonal-flow levels compared to the ITER-like case are identified.
\end{abstract}

(c) 2014 The Japan Society of Plasma Science and Nuclear Fusion Research

Keywords: gyrokinetic Vlasov simulation, realistic geometry, ITG mode

DOI: $10.1585 /$ pfr.9.1403029

\section{Introduction}

Turbulent transport of heat, particle, and momentum is a dominant transport channel in magnetically confined plasmas [1]. Understanding physics behind the turbulent transport and predicting quantitatively the resultant transport levels are, thus, important issues for the establishment of self-ignited steady burning plasmas in future fusion devices such as ITER and DEMO reactors. To this end, ion temperature gradient (ITG) driven turbulence, which is expected as one of the main causes of ion heat transport, has extensively been studied by means of gyrokinetic simulations [e.g., see Ref.[2]]. A number of important results on the ITG turbulent transport processes have been obtained so far, and the ion heat transport suppression by nonlinearly generated zonal flows is one of the most important findings $[3,4]$. In particular, local fluxtube gyrokinetic model [5], which is utilized for investigation of local micro-instabilities, fine-scale turbulence structures and the related transport levels and mechanisms, is nowadays a fundamental tool not only for theoretical studies, but also for experimental analyses.

In view of turbulence control, the plasma shaping is an effective method to improve the confinement performance in tokamaks. In addition to MHD stability, micro-

author's e-mail: nakata.motoki@jaea.go.jp instabilities, turbulent transport, and zonal-flow properties can be strongly affected by the plasma shape, such as elongation, triangularity and up-down asymmetry, through the change of the linear frequencies, mode-structures, and trapped/passing guiding-center orbits. There are some earlier works looking into shaping effects on microinstabilities and turbulent transport. Miller's analytic local equilibrium model [6] is widely used in the local fluxtube model, then the fundamental dependencies with respect to the elongation and triangularity parameters have been investigated [7-9]. The impact of negative triangularity on electron heat transport has also been investigated by using both global and local turbulence simulations $[10,11]$, where the change of electron precession drifts leads to the stabilization of trapped electron modes (TEM). Many efforts have also been devoted for the crosscode benchmark [12] and validation against experimental observations [13, 14].

The plasma shaping can also affect the zonal-flow dynamics. Especially, an extension of linear zonal-flow theory [15] has revealed that the residual zonal-flow level is enhanced due to the plasma elongation [16]. The residual zonal-flow theory including the plasma shaping has also been applied to gyrofluid simulations [17] based on kinetic closure model [18].

In our previous studies $[19,20]$ using a local fluxtube 
gyrokinetic Vlasov code GKV [21], a critical role of zonal flows leading to successive nonlinear entropy transfer processes in the regulated ITG turbulent transport has been elucidated, where a simple concentric circular tokamak geometry was considered. In this study, a realistic tokamak equilibrium calculated by a free-boundary 2D GradShafranov equation solver MEUDAS [22] is newly incorporated to GKV using an interface code IGS, in order to extend the linear and nonlinear analyses to the tokamak experimental conditions. The extension is also indispensable for the quantitative validation against experimental results and for the prediction studies towards future fusion devices. By using the extended code, linear gyrokinetic simulations are performed for two types of equilibria, i.e., ITER-like and highly-shaped cases, which are expected in JT-60SA tokamak device, where the MHD stability has already been well investigated [23]. Then, the ITG-mode stability and the residual zonal-flow levels are compared in detail. These linear results provide one with fundamental insights for the later nonlinear gyrokinetic simulation studies towards JT-60SA and ITER.

The rest of the paper is organized as follows. In Sec. 2, the interface code IGS is presented, as well as some basic introductions to flux coordinate systems. Then, calculation model in GKV and verification results are given in Sec. 3. In Sec. 4, linear simulation results on ITG-mode stability and residual zonal flows for JT-60SA plasmas are presented. Finally, the summary is given in Sec. 5 .

\section{Realistic Tokamak Geometry}

All the flux coordinates considered here are constructed by $2 \mathrm{D}$ data obtained from a free-boundary GradShafranov equation solver MEUDAS [22], where general up-down asymmetric tokamak equilibria with the divertor legs are calculated consistently with the realistic external coil configurations. The interface code connecting the flux coordinates to the fluxtube one used in local gyrokinetic simulations is briefly presented in this section.

\subsection{Magnetic field representation}

General expressions for the magnetic field $\boldsymbol{B}$ and the current density $\boldsymbol{j}$, as well as the ideal MHD force balance relation, in a flux coordinate system $(\rho, \theta, \zeta)$ are given by [24]

$$
\begin{aligned}
\boldsymbol{B} & =\Psi^{\prime} \nabla \zeta \times \nabla \rho+\Phi^{\prime} \nabla \rho \times \nabla \theta+\nabla \rho \times \nabla \tilde{\alpha} \\
& =\tilde{\beta} \nabla \rho+I \nabla \theta+J \nabla \zeta+\nabla \tilde{\gamma}, \\
\frac{4 \pi}{c} \boldsymbol{j} & =J^{\prime} \nabla \rho \times \nabla \zeta+I^{\prime} \nabla \rho \times \nabla \theta+\nabla \tilde{\beta} \times \nabla \rho, \\
p^{\prime} & =-I^{\prime} \boldsymbol{B} \cdot \nabla \theta-J^{\prime} \boldsymbol{B} \cdot \nabla \zeta+\boldsymbol{B} \cdot \nabla \tilde{\beta},
\end{aligned}
$$

where $\rho$ is the radial coordinate representing a magnetic flux surface, and $\theta$ and $\zeta$ are the poloidal- and toroidalangle coordinates, respectively. The prime symbol means the $\rho$-derivative: $X^{\prime}=\mathrm{d} X(\rho) / \mathrm{d} \rho$, and $c$ denotes the speed of light. In addition to the contravariant representation for
$\boldsymbol{B}$ as in Eq. (1), the covariant one is shown in Eq. (2). The toroidal magnetic flux, the poloidal one, and the toroidal current inside the magnetic flux surface $\rho$ are denoted by $2 \pi \Phi, 2 \pi \Psi$ and $2 \pi I$, respectively, while $2 \pi J$ represents the poloidal current outside $\rho$. Ensuring physical singlevaluedness for $\boldsymbol{B}$ and $\boldsymbol{j}$, the quantities $\tilde{\alpha}(\rho, \theta, \zeta), \tilde{\beta}(\rho, \theta, \zeta)$, and $\tilde{\gamma}(\rho, \theta, \zeta)$ must be doubly periodic functions with respect to $\theta$ and $\zeta$. It should be noted that Eqs. (1) - (4) are of invariance under the following transformation,

$$
\begin{aligned}
& \tilde{\alpha}^{*}=\tilde{\alpha}+g(\rho), \\
& \tilde{\beta}^{*}=\tilde{\beta}+h^{\prime}(\rho), \\
& \tilde{\gamma}^{*}=\tilde{\gamma}-h(\rho),
\end{aligned}
$$

where $g$ and $h$ are arbitrary flux functions depending only on $\rho$, and note that $\tilde{\beta}$ and $\tilde{\gamma}$ should be simultaneously transformed by $h$. The Jacobian $\sqrt{g}_{\rho \theta \zeta}$ and the flux-surfaceaverage operator $\langle\cdots\rangle$ are defined as

$$
\begin{aligned}
\sqrt{g}_{\rho \theta \zeta} & =(\nabla \rho \times \nabla \theta \cdot \nabla \zeta)^{-1}, \\
\langle X\rangle & =\frac{1}{V^{\prime}} \iint_{0}^{2 \pi} \mathrm{d} \theta \mathrm{d} \zeta \sqrt{g}_{\rho \theta \zeta} X(\rho, \theta, \zeta), \\
V^{\prime} & =\iint_{0}^{2 \pi} \mathrm{d} \theta \mathrm{d} \zeta \sqrt{g}_{\rho \theta \zeta},
\end{aligned}
$$

where $V^{\prime}(\rho)$ is the radial derivative of the plasma volume at $\rho$. Solvability constraints of the magnetic differential equations, i.e., $\langle\boldsymbol{B} \cdot \nabla \tilde{\gamma}\rangle=0$ and $\langle\boldsymbol{B} \cdot \nabla \tilde{\beta}\rangle=0$ in Eqs. (1), (2) and (4), provide one with

$$
\begin{aligned}
\left\langle B^{2}\right\rangle & =4 \pi^{2} \frac{\Phi^{\prime}}{V^{\prime}}\left(J+q^{-1} I\right), \\
p^{\prime} & =-4 \pi^{2} \frac{\Phi^{\prime}}{V^{\prime}}\left(J^{\prime}+q^{-1} I^{\prime}\right),
\end{aligned}
$$

where $q(\rho)=\Phi^{\prime} / \Psi^{\prime}$ denotes the safety factor profile.

Although the above relations are consequence of general discussions, irrespective of the choice of $(\rho, \theta, \zeta)$, one can construct more specific coordinate systems, which are useful for theoretical and/or numerical analyses. Following Pustovitov's method [25], the most important three fluxcoordinate systems, i.e., Hamada, Boozer, and axisymmetric (or natural) coordinates, are classified systematically.

First, from the definition of the flux-surface average [Eq. (9)], one finds that the Jacobian can be written by an arbitrary function $f=f(\rho, \theta, \zeta)$,

$$
\frac{1}{\sqrt{g}_{\rho \theta \zeta}}=\frac{4 \pi^{2}}{V^{\prime}} \frac{f}{\langle f\rangle} .
$$

Note that the physical dimension of $f$ is irrelevant in the above definition, and this is significant feature to define various coordinates in the same manner. The above relation is applied to Eq. (1), then we find

$$
\begin{aligned}
& (\boldsymbol{B}-\nabla \rho \times \nabla \tilde{\alpha}) \cdot \nabla \theta=4 \pi^{2} \frac{\Psi^{\prime}}{V^{\prime}} \frac{f}{\langle f\rangle}, \\
& (\boldsymbol{B}-\nabla \rho \times \nabla \tilde{\alpha}) \cdot \nabla \zeta=4 \pi^{2} \frac{\Phi^{\prime}}{V^{\prime}} \frac{f}{\langle f\rangle} .
\end{aligned}
$$


Equations (14) and (15) are coupled equations with respect to four variables, $\tilde{\alpha}, f$ (or $\sqrt{g}_{\rho \theta \zeta}$ ), $\theta$, and $\zeta$, for given magnetic field $\boldsymbol{B}$ and the radial coordinate $\rho$. When any two of them are given, one can obtain the corresponding flux coordinate system determined by Eqs. (14) and (15). Then, Hamada, Boozer, and the axisymmetric coordinates are classified as follows:

$$
\begin{aligned}
& \text { Hamada }\left(\rho, \theta_{H}, \zeta_{H}\right): \tilde{\alpha}=0, f=\text { Const., } \\
& \text { Boozer }\left(\rho, \theta_{B}, \zeta_{B}\right): \tilde{\alpha}=0, f=B^{2},
\end{aligned}
$$

Axisymmetric $\left(\rho, \theta_{A}, \zeta_{A}\right): \tilde{\alpha}=0, f=\boldsymbol{B} \cdot \nabla \zeta_{A}, \zeta_{A}=\phi$,

where $\tilde{\alpha}=0$ makes the magnetic field line straight, i.e., $\mathrm{d} \zeta / \mathrm{d} \theta=\boldsymbol{B} \cdot \nabla \zeta / \boldsymbol{B} \cdot \nabla \theta=q(\rho)$, then such coordinates are called the straight-field-line (SFL) flux coordinates. Rigorously speaking, $\tilde{\alpha}=s(\rho)$ ( $s$ is an arbitrary flux function) also gives the SFL flux coordinates, but one can always set $s(\rho)$ to be 0 by Eq. (5). Note also that the second condition in Eq. (18), $f=\boldsymbol{B} \cdot \nabla \zeta_{A}$, is actually equivalent to the flux-surface average of Eq. (15), and does not provide any relevant equations for determining $\zeta$. Thus, $\zeta_{A}=\phi$ should be imposed as an additional condition for the axisymmetric coordinates, where $\phi$ denotes the azimuthal angle in the cylindrical coordinates $(R, Z, \phi)$. Each coordinate system possesses different advantages, and their detailed properties and the components of $\boldsymbol{B}$ and $\boldsymbol{j}$ are summarized in Appendix A.

The coordinate transformation from one to another is given by the generating function $G(\rho, \theta, \zeta)$ :

$$
\begin{aligned}
& \bar{\theta}=\theta+\Psi^{\prime} G(\rho, \theta, \zeta), \\
& \bar{\zeta}=\zeta+\Phi^{\prime} G(\rho, \theta, \zeta),
\end{aligned}
$$

where $G$ is determined by the following magnetic differential equation,

$$
\boldsymbol{B} \cdot \nabla G(\rho, \theta, \zeta)=\frac{1}{\sqrt{g}_{\rho \bar{\theta} \bar{\zeta}}}-\frac{1}{\sqrt{g}_{\rho \theta \zeta}} .
$$

For instance, $G\left(\rho, \theta_{B}, \zeta_{B}\right)$ giving the transformation from Hamada to Boozer coordinates is

$$
G\left(\rho, \theta_{B}, \zeta_{B}\right)=\frac{\left\langle B^{2}\right\rangle}{\Phi^{\prime} p^{\prime}\left(J+q^{-1} I\right)} \tilde{\beta}^{*}\left(\rho, \theta_{B}, \zeta_{B}\right) .
$$

\subsection{Numerical constructions of SFL flux co- ordinates}

The free-boundary Grad-Shafranov equation solver MEUDAS provides one with 2D data of $\Psi(R, Z)$ for given $p(\Psi)$ and $J(\Psi)$. These fundamental data are, then, input to a newly developed interface code IGS, which produces Hamada, Boozer, and the axisymmetric coordinates numerically. The basic idea in IGS is similar to that in Ref. [26]. In addition to the original data format in MEUDAS, IGS can also treat the G-EQDSK format which is widely used in the fusion physics field. The radial coordinate is chosen as $\rho=\left(\Psi / \Psi_{\text {edge }}\right)^{1 / 2}$ or $\rho=\left(\Phi / \Phi_{\text {edge }}\right)^{1 / 2}$, where $\Psi_{\text {edge }}$ and $\Phi_{\text {edge }}$ are the poloidal and toroidal magnetic fluxes at the plasma edge, respectively. In IGS, the cubic-spline interpolation is applied to the $2 \mathrm{D}$ rectangular data of $\Psi(R, Z)$. Then, the axisymmetric coordinates, $R=R\left(\rho, \theta_{A}\right), Z=Z\left(\rho, \theta_{A}\right), \zeta_{A}=\phi$, are first constructed from $\Psi(R, Z)$ by solving $\mathrm{d} R / \mathrm{d} l_{\mathrm{p}}=|\nabla \Psi|^{-1} \partial \Psi / \partial Z$, $\mathrm{d} Z / \mathrm{d} l_{\mathrm{p}}=-|\nabla \Psi|^{-1} \partial \Psi / \partial R, \mathrm{~d} \theta_{A} / \mathrm{d} l_{\mathrm{p}}=J / q R|\nabla \Psi|$, where $\mathrm{d} l_{\mathrm{p}}$ is the arc-length element on the poloidal cross section. After that, Hamada and Boozer coordinates, $R=R\left(\rho, \theta_{H, B}\right)$, $Z=Z\left(\rho, \theta_{H, B}\right), \zeta_{H, B}=\phi+\Phi^{\prime} G_{H, B}\left(\rho, \theta_{A}\right)$ are obtained from Eqs. (19) - (21).

The covariant metric tensor, $g_{i j}$ is calculated by the following relation:

$$
g_{i j}=\frac{\partial R}{\partial u_{i}} \frac{\partial R}{\partial u_{j}}+\frac{\partial Z}{\partial u_{i}} \frac{\partial Z}{\partial u_{j}}+R^{2} \frac{\partial \phi}{\partial u_{i}} \frac{\partial \phi}{\partial u_{j}},
$$

where $\left(u_{i}, u_{j}\right)=\{\rho, \theta, \zeta\}$ (the coordinates label $A, B$, and $H$ are omitted). The determinant of $g_{i j}$ gives the Jacobian $\sqrt{g}_{\rho \theta \zeta}=\operatorname{det}\left(g_{i j}\right)^{1 / 2}$, and the contravariant metric tensor $g^{i j}$ is calculated by

$$
g^{i l}=\frac{1}{\sqrt{g}_{\rho \theta \zeta}^{2}}\left(g_{j m} g_{k n}-g_{j n} g_{k m}\right),
$$

where $\{i, j, k\}$ and $\{l, m, n\}$ are the even permutations of $\{\rho, \theta, \zeta\}$.

The examples of constructed flux coordinates are shown in Fig. 1. Here, the Solov'ev analytic equilibrium
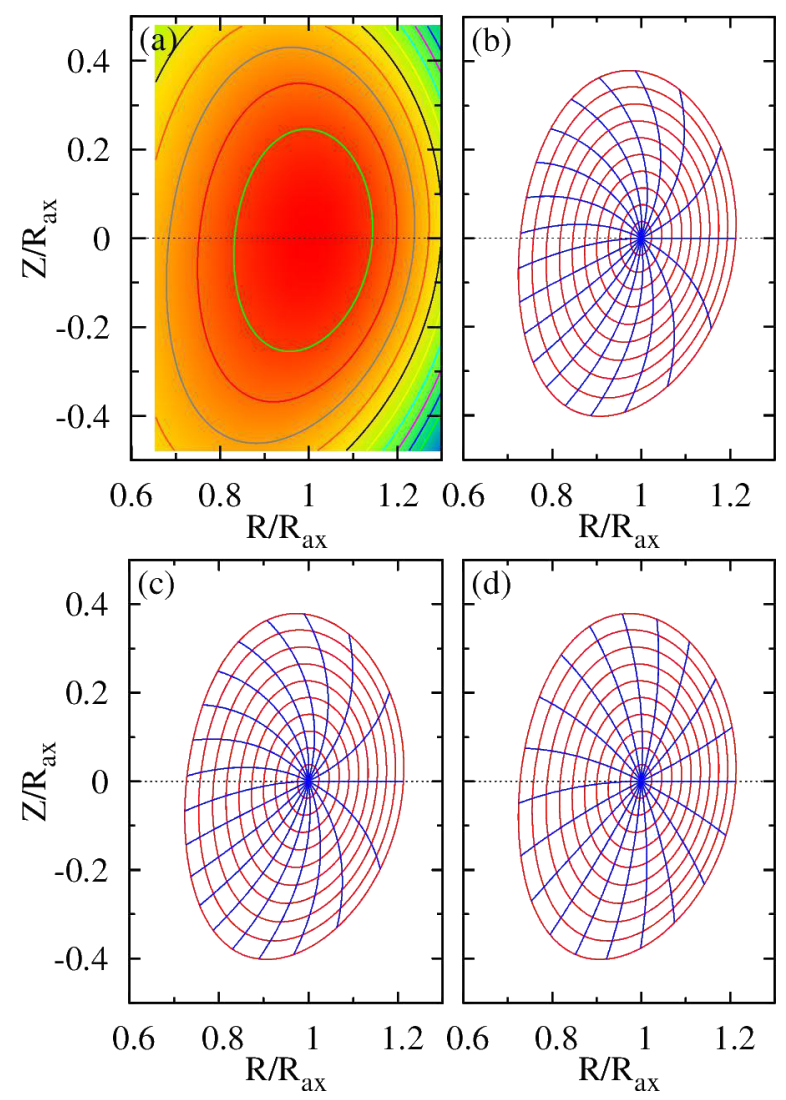

Fig. 1 Comparison of the SFL coordinates: (a) the original $2 \mathrm{D}$ data $\Psi(R, Z)$ for the Solov'ev analytic equilibrium with the up-down asymmetry, and (b) axisymmetric, (c) Boozer, (d) Hamada coordinates constructed by IGS. 
with up-down asymmetry is considered:

$$
\begin{aligned}
\frac{\Psi}{\Psi_{\text {edge }}}= & \frac{1}{\epsilon_{a}^{2}}\left[\frac{(1-D) \bar{R}^{2} \bar{Z}^{2}-D \bar{Z}^{2}}{E^{2}}+\frac{1}{4}\left(\bar{R}^{2}-1\right)^{2}\right] \\
& +\frac{\nu_{\text {asym }}}{\epsilon_{a}}\left(\bar{R}^{2}-1\right) \bar{Z},
\end{aligned}
$$

where $\bar{R}$ and $\bar{Z}$ are the positions normalized by the major radius on the magnetic axis $R_{\mathrm{ax}}$, and $\epsilon_{a}=a / R_{\mathrm{ax}}, E, D$, and, $v_{\text {asym }}$ are shape parameters related to the inverse aspect ratio, elongation, triangularity, and up-down asymmetry, respectively. One finds that the iso-angle surfaces of $\theta$ in Hamada coordinates are less deformed in comparison with those in Boozer and the axisymmetric ones. Instead, in the non-axisymmetric systems, Hamada coordinates show relatively strong deformation of iso-angle surfaces of $\zeta$ which leads to the spectral broadening on $B$ in the toroidal mode numbers [24].

A numerical accuracy of the constructed flux coordinates is verified by comparisons with the analytic expression. Figure 2 shows the absolute errors indicating the deviation from the following analytic relations,

$$
\begin{aligned}
\frac{1}{\sqrt{g}_{\rho \theta \zeta} \frac{\partial R[\rho, \theta]}{\partial \theta}} & =-\frac{1}{R} \frac{\partial \rho[R, Z]}{\partial Z}, \\
g^{\rho \rho}[\rho, \theta] & =\left|\frac{\partial \rho[R, Z]}{\partial R} \nabla R+\frac{\partial \rho[R, Z]}{\partial Z} \nabla Z\right|^{2},
\end{aligned}
$$

where $\rho=\left(\Psi / \Psi_{\text {edge }}\right)^{1 / 2}$ is considered here.
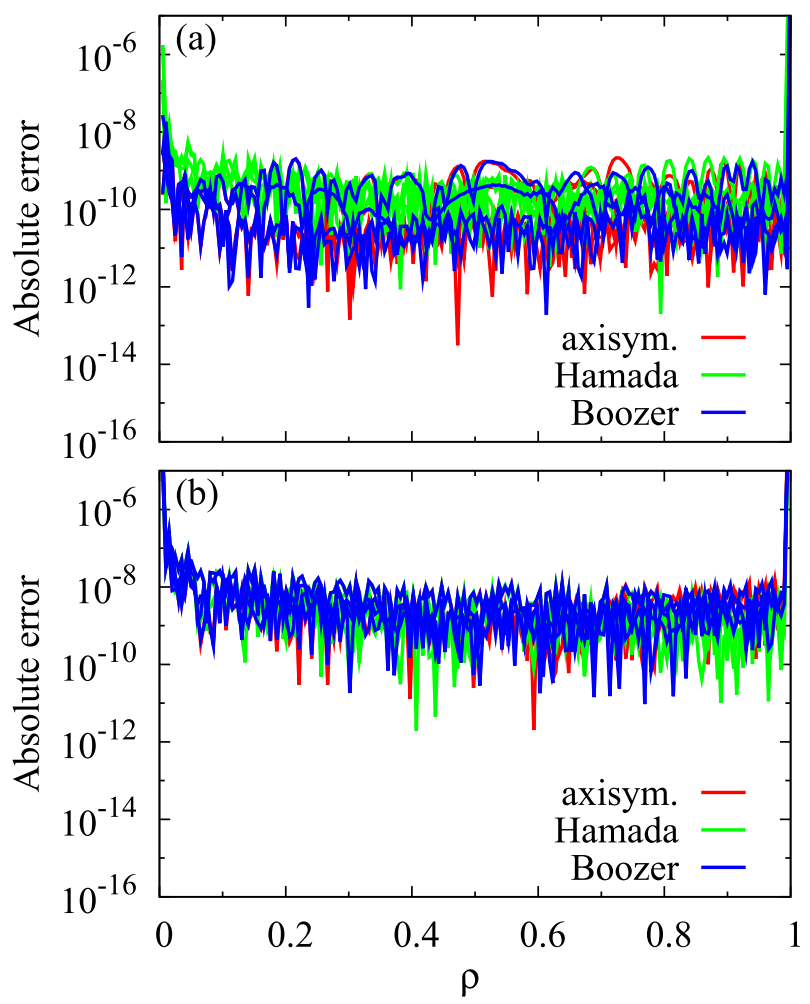

Fig. 2 Comparison of the absolute errors in (a) Eq. (26) and (b) Eq. (27), for axisymmetric, Hamada, and Boozer coordinates, where the radial distributions for $\theta_{A, H, B}=$ $\{0, \pi / 2, \pi, 3 \pi / 2\}$ are plotted in each coordinate system.
One can see that all the constructed flux coordinates indicate the numerical accuracy up to $\sim 10^{-8}$. Besides, the consistency among the metric tensor and magnetic field components, e.g., $B_{\theta}=B^{\theta} g_{\theta \theta}+B^{\zeta} g_{\zeta \theta}$, have been confirmed in IGS.

\subsection{Local fluxtube coordinate system}

Local fluxtube coordinates $(x, y, z)$ used in gyrokinetic simulations are based on the SFL flux coordinates. The definition of each coordinate is given as

$$
x=c_{x}\left(\rho-\rho_{0}\right), y=c_{y}[q(\rho) \theta-\zeta], z=\theta,
$$

where $(\rho, \theta, \zeta)$ are arbitrary SFL flux coordinates, and $c_{x}$, $c_{y}$, and $\rho_{0}$ are the normalization factors and the reference radial position, respectively. Using the above definitions, the magnetic field $\boldsymbol{B}$ is written as

$$
\boldsymbol{B}=c_{B} \nabla x \times \nabla y, \quad c_{B}=\Psi^{\prime} c_{x}^{-1} c_{y}^{-1} .
$$

The partial-derivative operators with respect to $x, y$, and $z$ are given by

$$
\begin{aligned}
\frac{\partial}{\partial x} & =\frac{1}{c_{x}} \frac{\partial}{\partial \rho}+\frac{q \hat{s} \theta}{c_{x} \rho} \frac{\partial}{\partial \zeta}, \\
\frac{\partial}{\partial y} & =-\frac{1}{c_{y}} \frac{\partial}{\partial \zeta}, \\
\frac{\partial}{\partial z} & =\frac{\partial}{\partial \theta}+q \frac{\partial}{\partial \zeta},
\end{aligned}
$$

where $\hat{s}=\rho q^{\prime} / q$ denotes the magnetic shear. Note that $\partial / \partial y=0$ for axisymmetric plasmas with $\partial / \partial \zeta=0$. One can also obtain the contravariant metric tensor and the Jacobian as follows:

$$
\begin{aligned}
g^{x x}= & \nabla x \cdot \nabla x=c_{x}^{2} g^{\rho \rho}, \\
g^{x y}= & \nabla x \cdot \nabla y=c_{x} c_{y}\left[\left(\frac{q \hat{s} \theta}{\rho}\right) g^{\rho \rho}+q g^{\rho \theta}-g^{\rho \zeta}\right], \\
g^{x z}= & \nabla x \cdot \nabla z=c_{x} g^{\rho \theta}, \\
g^{y y}= & \nabla y \cdot \nabla y=c_{y}^{2}\left[\left(\frac{q \hat{s} \theta}{\rho}\right)^{2} g^{\rho \rho}+q^{2} g^{\theta \theta}+g^{\zeta \zeta}\right. \\
& \left.+\left(\frac{2 q^{2} \hat{s} \theta}{\rho}\right) g^{\rho \theta}-2 q g^{\theta \zeta}-\left(\frac{2 q \hat{s} \theta}{\rho}\right) g^{\rho \zeta}\right], \\
g^{y z}= & \nabla y \cdot \nabla z=c_{y}\left[\left(\frac{q \hat{s} \theta}{\rho}\right) g^{\rho \theta}+q g^{\theta \theta}-g^{\theta \zeta}\right], \\
g^{z z}= & \nabla z \cdot \nabla z=g^{\theta \theta}, \\
\sqrt{g}_{x y z}= & (\nabla x \times \nabla y \cdot \nabla z)^{-1}=c_{x}^{-1} c_{y}^{-1} \sqrt{g}_{\rho \theta \zeta} .
\end{aligned}
$$

The wavenumber vector perpendicular to the magnetic field and the differential operator along the field line are defined by

$$
\begin{aligned}
\boldsymbol{k}_{\perp} & =k_{x} \nabla x+k_{y} \nabla y, \\
k_{\perp}^{2} & =k_{x}^{2} g^{x x}+2 k_{x} k_{y} g^{x y}+k_{y}^{2} g^{y y}, \\
\boldsymbol{b} \cdot \nabla & =\frac{c_{B}}{B \sqrt{g}_{x y z}} \frac{\partial}{\partial z},
\end{aligned}
$$

where $\boldsymbol{b}$ is the unit vector parallel to the magnetic field. 
As will be shown in Sec. 3, the radial coordinate of $\rho=\left(\Phi / \Phi_{\text {edge }}\right)^{1 / 2}$ and $c_{x}=a, c_{y}=a \rho_{0} / q\left(\rho_{0}\right), c_{B}=B_{\text {ax }}$ are basically used in GKV, where $B_{\text {ax }}$ denotes the magnetic field intensity on the magnetic axis of $\rho=0$, and the plasma minor radius is defined by $a=\left(2 \Phi_{\text {edge }} / B_{\text {ax }}\right)^{1 / 2}$. Note that, in the local fluxtube formulation shown below, all the normalized quantities and operators do not depend explicitly on the plasma minor radius $a$, but on the inverse aspect ratio $\epsilon_{a}$. Although IGS gives us the complete metric information of realistic equilibria from MEUDAS, the analytic geometry model is still useful to subtract the shaping effects. The analytic expression of the concentric circular equilibrium model and the simplified $s-\alpha$ one are summarized in Appendix B.

\section{Local Gyrokinetic Vlasov Simula- tion Code GKV}

In this section, we present the simulation model implemented in GKV, as well as the verification and benchmark test with realistic geometry from the MHD equilibrium solver.

\subsection{Calculation model}

Calculation model in GKV is briefly summarized below including electromagnetic effects. One can also find the implementation of VMEC equilibria for 3dimensional helical/stellalator plasmas in Ref. [27]. The wavenumber-space representation of electromagnetic gyrokinetic Vlasov equation for the particle species of $s$ is given by

$\left(\frac{\partial}{\partial t}+v_{\|} \boldsymbol{b} \cdot \nabla+i \omega_{\mathrm{Ds}}\right) \delta h_{\mathrm{s} \boldsymbol{k}_{\perp}}-\frac{c}{B} \sum_{\Delta} \boldsymbol{b} \cdot\left(\boldsymbol{k}_{\perp}^{\prime} \times \boldsymbol{k}_{\perp}^{\prime \prime}\right) \delta \psi_{\mathrm{s} \boldsymbol{k}_{\perp}^{\prime}} \delta h_{\mathrm{s} \boldsymbol{k}_{\perp}^{\prime \prime}}$

$=\frac{e_{\mathrm{s}} F_{\mathrm{Ms}}}{T_{\mathrm{s}}}\left(\frac{\partial}{\partial t}+i \omega_{* T \mathrm{~s}}\right) \delta \psi_{\mathrm{s} k_{\perp}}+C_{\mathrm{s}}\left(\delta h_{\mathrm{s} k_{\perp}}\right)$,

where $\delta h_{\mathrm{s} k_{\perp}}=\delta h_{\mathrm{s} k_{\perp}}\left(z, v_{\|}, \mu\right)$ stands for the non-adiabatic part of the gyrocenter perturbed distribution function $\delta f_{\mathrm{s} \boldsymbol{k}_{\perp}}^{(\mathrm{g})}$, i.e., $\delta h_{\mathrm{s} \boldsymbol{k}_{\perp}}=\delta f_{\mathrm{s} \boldsymbol{k}_{\perp}}^{(\mathrm{g})}+e_{\mathrm{s}} J_{0}\left(k_{\perp} v_{\perp} / \Omega_{\mathrm{s}}\right) \delta \phi_{\boldsymbol{k}_{\perp}} F_{\mathrm{Ms}} / T_{\mathrm{s}}$, and $m_{\mathrm{s}}$, $e_{\mathrm{s}}, T_{\mathrm{s}}$, and $\Omega_{\mathrm{s}}=e_{\mathrm{s}} B / m_{\mathrm{s}} c$ are the particle mass, the electric charge, the equilibrium temperature, and the gyrofrequency, respectively. The parallel velocity $v_{\|}$and the magnetic moment $\mu$ are used as the velocity-space coordinates, where $\mu$ is defined by $\mu \equiv m_{\mathrm{s}} v_{\perp}^{2} / 2 B$ with the perpendicular velocity $v_{\perp}$. The potential fluctuations averaged over the gyrophase are denoted by $\delta \psi_{\mathrm{s} \boldsymbol{k}_{\perp}}=J_{0}\left(k_{\perp} v_{\perp} / \Omega_{\mathrm{s}}\right)\left[\delta \phi_{\boldsymbol{k}_{\perp}}-\right.$ $\left(v_{\|} / c\right) \delta A_{\| \boldsymbol{k}_{\perp}}$, where $J_{0}$ is the zeroth-order Bessel function, and the former and latter terms mean the electrostatic and electromagnetic parts. Since we often focus on the finite but low- $\beta$ plasmas, the parallel magnetic field fluctuation $\delta B_{\| k_{\perp}}$ is ignored here. The equilibrium part of the distribution function is given by the local Maxwellian distribution, i.e., $F_{\mathrm{Ms}}=n_{\mathrm{s}}\left(m_{\mathrm{s}} / 2 \pi T_{\mathrm{s}}\right)^{3 / 2} \exp \left[-\left(m_{\mathrm{s}} v_{\|}^{2}+2 \mu B\right) / 2 T_{\mathrm{s}}\right]$, where $n_{\mathrm{s}}$ represents the equilibrium density. The symbol $\Sigma_{\Delta}$ appearing in the nonlinear term of Eq. (43) means double summations with respect to $\boldsymbol{k}_{\perp}^{\prime}$ and $\boldsymbol{k}_{\perp}^{\prime \prime}$, which satisfy the triad-interaction condition of $\boldsymbol{k}_{\perp}=\boldsymbol{k}_{\perp}^{\prime}+\boldsymbol{k}_{\perp}^{\prime \prime}$. Collisional effects are introduced in terms of a linearized model collision operator $C_{\mathrm{s}}$.

The magnetic and diamagnetic drift frequencies, $\omega_{\text {Ds }}$ and $\omega_{* T \mathrm{~s}}$, are given by

$$
\begin{aligned}
\omega_{\text {Ds }} & =\frac{c T_{\mathrm{s}}}{e_{\mathrm{s}} B} \boldsymbol{k}_{\perp} \cdot \boldsymbol{b} \times\left(\mu \nabla B+m_{\mathrm{s}} v_{\|}^{2} \boldsymbol{b} \cdot \nabla \boldsymbol{b}\right) \\
& =\frac{c\left(m_{\mathrm{s}} v_{\|}^{2}+\mu B\right)}{e_{\mathrm{s}} c_{B}}\left(\mathcal{K}_{x} k_{x}+\mathcal{K}_{y} k_{y}\right), \\
\omega_{* T \mathrm{~s}} & =\frac{c T_{\mathrm{s}}}{e_{\mathrm{s}} B}\left[1+\eta_{\mathrm{s}}\left(\frac{m_{\mathrm{s}} v_{\|}^{2}+2 \mu B}{2 T_{\mathrm{s}}}-\frac{3}{2}\right)\right] \boldsymbol{k}_{\perp} \cdot \boldsymbol{b} \times \nabla \ln n_{\mathrm{s}} \\
& =-\frac{c T_{\mathrm{s}}}{e_{\mathrm{s}} c_{B}}\left[\frac{1}{L_{n_{\mathrm{s}}}}+\frac{1}{L_{T_{\mathrm{s}}}}\left(\frac{m_{\mathrm{s}} v_{\|}^{2}+2 \mu B}{2 T_{\mathrm{s}}}-\frac{3}{2}\right)\right] k_{y},
\end{aligned}
$$

where $\eta_{\mathrm{s}}=L_{n_{\mathrm{s}}} / L_{T_{\mathrm{s}}}$ with $L_{n_{\mathrm{s}}}=-\left(\mathrm{d} \ln n_{\mathrm{s}} / \mathrm{d} x\right)^{-1}$ and $L_{T_{\mathrm{s}}}=$ $-\left(\mathrm{d} \ln T_{\mathrm{s}} / \mathrm{d} x\right)^{-1}$. The geometric coefficients $\mathcal{K}_{x}$ and $\mathcal{K}_{y}$ are defined as follows:

$$
\begin{aligned}
& \mathcal{K}_{x}=\frac{g^{x z} g^{x y}-g^{x x} g^{y z}}{B^{2} / c_{B}^{2}} \frac{\partial \ln B}{\partial z}-\frac{\partial \ln B}{\partial y}, \\
& \mathcal{K}_{y}=\frac{g^{x z} g^{y y}-g^{x y} g^{y z}}{B^{2} / c_{B}^{2}} \frac{\partial \ln B}{\partial z}+\frac{\partial \ln B}{\partial x} .
\end{aligned}
$$

The electromagnetic potential fluctuations are determined by the Poisson-Ampère equations:

$$
\begin{aligned}
& \left(k_{\perp}^{2}+\lambda_{\mathrm{D}}^{-2}\right) \delta \phi_{k_{\perp}}=4 \pi \sum_{\mathrm{s}} e_{\mathrm{s}} \int \mathrm{d} v J_{0 \mathrm{~s}}\left(k_{\perp} v_{\perp} / \Omega_{\mathrm{s}}\right) \delta h_{\mathrm{s} k_{\perp}}, \\
& k_{\perp}^{2} \delta A_{\| k_{\perp}}=\frac{4 \pi}{c} \sum_{\mathrm{s}} e_{\mathrm{s}} \int \mathrm{d} v v_{\|} J_{0 \mathrm{~s}}\left(k_{\perp} v_{\perp} / \Omega_{\mathrm{s}}\right) \delta h_{\mathrm{s} k_{\perp}},
\end{aligned}
$$

where $\lambda_{\mathrm{D}}=\left(\sum_{\mathrm{s}} 4 \pi n_{\mathrm{s}} e_{\mathrm{s}}^{2} / T_{\mathrm{s}}\right)^{-1 / 2}$ is the Debye length. Note that, in the adiabatic electron limit with $k_{\perp} \rho_{\text {te }} \ll 1$, the gyrocenter perturbed distribution for electrons is approximated by $\int \mathrm{d} \boldsymbol{v} \delta f_{\mathrm{e} \boldsymbol{k}_{\perp}}^{\mathrm{(g)}} \simeq-e n_{\mathrm{e}}\left(\delta \phi_{\boldsymbol{k}_{\perp}}-\left\langle\delta \phi_{\boldsymbol{k}_{\perp}}\right\rangle_{z} \delta_{k_{y}, 0}\right) / T_{\mathrm{e}}$, where $\rho_{\mathrm{ts}}=m_{\mathrm{s}} c v_{\mathrm{ts}} /\left|e_{\mathrm{s}}\right| B_{\mathrm{ax}}$ means the gyroradius evaluated with the thermal speed $v_{\mathrm{ts}}=\left(T_{\mathrm{s}} / m_{\mathrm{s}}\right)^{1 / 2}$, and the field-line average is defined by $\left\langle X_{\boldsymbol{k}_{\perp}}\right\rangle_{z}=\int \mathrm{d} z \sqrt{g}_{x y z} X_{\boldsymbol{k}_{\perp}} / \int \mathrm{d} z \sqrt{g}_{x y z}$.

By using the above gyrokinetic Vlasov and Poisson Ampère equations, one can derive another important equation describing the balance and transfer of the entropy variable defined as $\delta S_{\mathrm{s} \boldsymbol{k}_{\perp}} \equiv\left\langle\int \mathrm{d} \boldsymbol{v}\left|\delta f_{\mathrm{s} \boldsymbol{k}_{\perp}}^{(\mathrm{g})}\right|^{2} / 2 F_{\mathrm{Ms}}\right\rangle_{z}$. The entropy balance equation [e.g., see Ref. [28]] provides us with many physical insights closely associated with the turbulence saturation, and is formulated with the relation among the development of fine-scale fluctuations on the distribution function, the turbulent transport, and the collisional dissipation, as well as the linear and nonlinear entropy transfer dynamics. The detailed numerical analyses of the entropy balance and the transfer processes in ITG turbulence are shown in, e.g., Ref. [19-21,29].

\subsection{Code verification and benchmark test}

The implementation of realistic MHD equilibria on GKV is verified by the benchmark test with other gyrokinetic codes, GENE [30], GS2 [31], and GKW [32]. In 


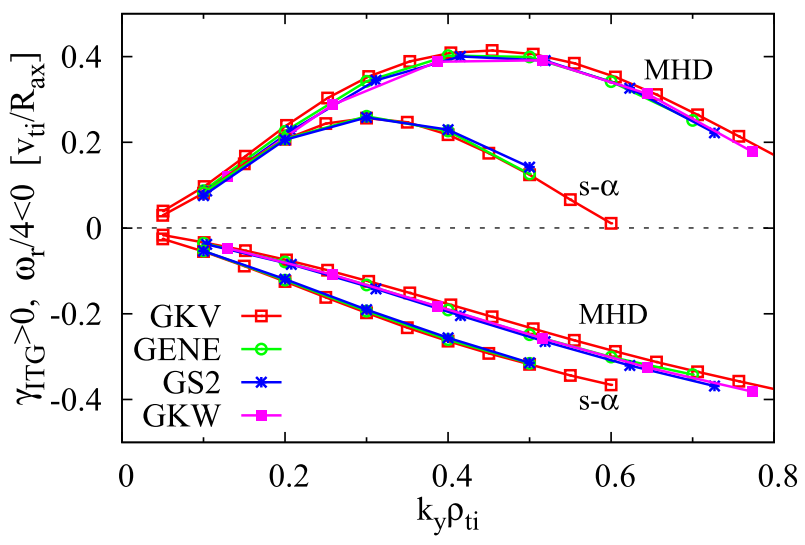

Fig. 3 Cross-code benchmark result on the ITG-mode dispersion relations with the analytic $s-\alpha$ equilibrium and Cyclone-base-case like MHD equilibrium produced by CHEASE.

this benchmark test, the same geometry data for Cyclonebase-case like MHD equilibrium from an MHD solver CHEASE [33] , which is used in Ref. [34], is also applied to GKV. The fluxtube geometry on the axisymmetric coordinates with $\rho=\left(\Psi / \Psi_{\text {edge }}\right)^{1 / 2}$ is considered here. The growth rate and the real frequency of electrostatic ITG modes with the adiabatic electrons are compared in Fig. 3, where the detailed physical parameters are shown in Ref. [34]. In addition to the realistic MHD equilibrium, the results with analytic $s-\alpha$ models are also compared. The implementation of the realistic MHD geometry to GKV and IGS has successfully been verified with good agreement in the dispersion relation of the ITG-mode.

\section{Linear Gyrokinetic Analyses with JT-60SA Tokamak Equilibria}

The extended GKV with IGS is applied to linear stability and residual zonal-flow analyses for the typical plasmas expected in JT-60SA tokamak device.

\subsection{ITER-like and highly-shaped plasmas}

In this study, two typical plasmas expected in JT60SA are considered. One is an ITER-like plasma with single-null separatrix [Figs. 4 (a) and 4(b)], and the other is the highly-shaped plasma with quasi-double-null separatrix [Figs. 4 (c) and 4(d)], where the basic equilibrium parameters are summarized in Table 1. The highly-shaped plasma shows higher plasma current $I_{\mathrm{p}}$, normalized $\beta \beta_{\mathrm{N}}$, elongation $\kappa$, and triangularity $\delta$. Besides, the global shaping factor $S=q_{95} I_{\mathrm{p}} / B_{\mathrm{ax}} a$ is 1.45 times larger than that in the ITER-like plasma, where $q_{95}=q(\rho=0.95)$. Note also that the highly-shaped plasma is almost up-down symmetric $\left(\kappa_{95}^{\text {up }} \simeq \kappa_{95}^{\text {down }}, \delta_{95}^{\text {up }} \simeq \delta_{95}^{\text {down }}\right)$, while the ITER-like plasma shows the up-down asymmetry ( $\left.\kappa_{95}^{\text {up }}<\kappa_{95}^{\text {down }}, \delta_{95}^{\text {up }}<\delta_{95}^{\text {down }}\right)$. The $q$ - and $\hat{s}$-profiles for both cases are plotted in Fig. 5. The ideal MHD stabilities have been well examined in
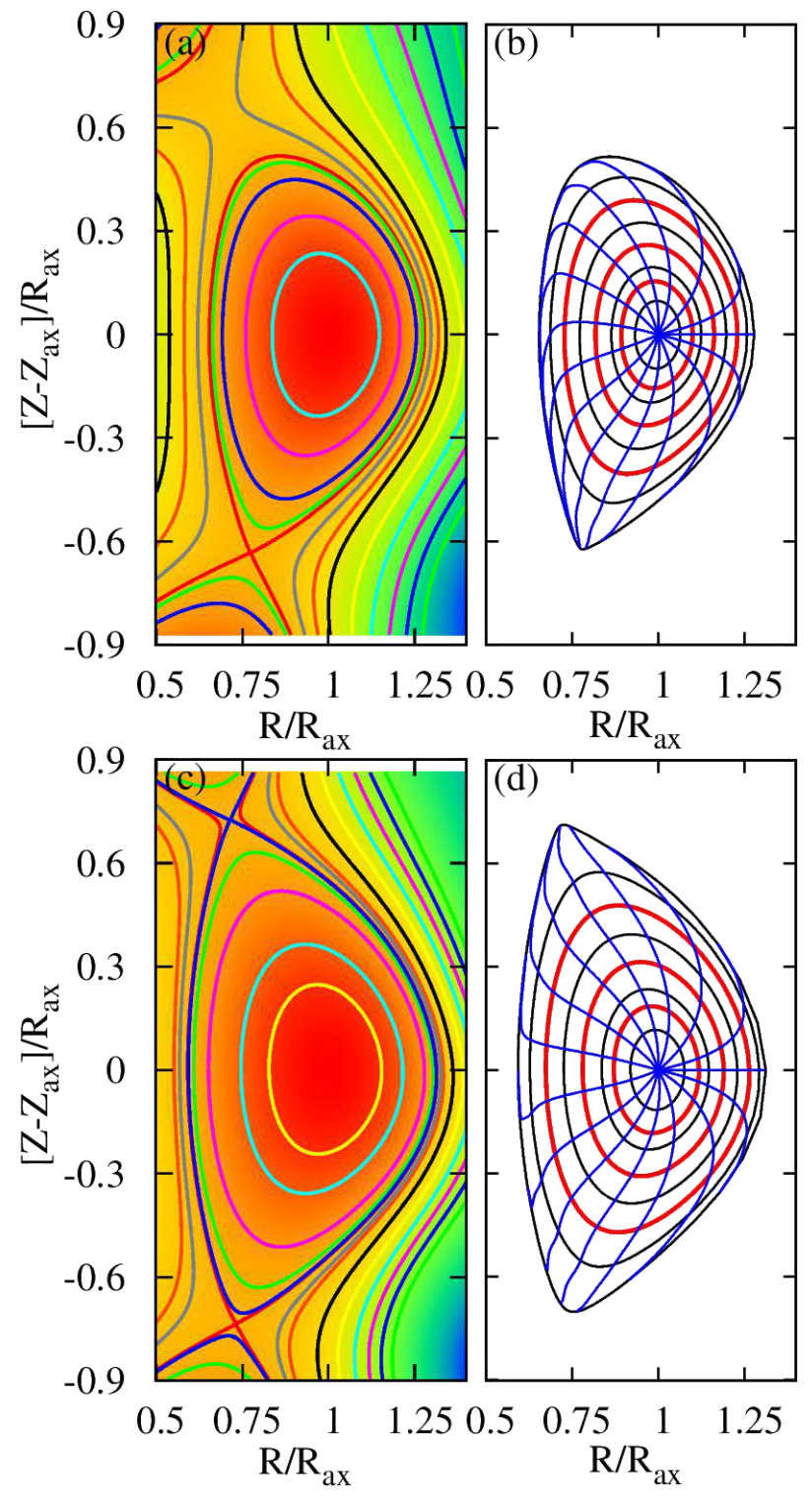

Fig. 4 Comparisons of the original $2 \mathrm{D}$ data $\Psi(R, Z)$ for (a) ITER-like and (b) highly-shaped equilibria produced by MEUDAS, and of [(b), (d)] constructed Boozer coordinates by IGS, where $Z_{\mathrm{ax}}$ means the vertical elevation of the magnetic axis. The flux surfaces for $\rho=$ $\{0.30,0.50,0.75\}$ are shown by red thick lines in (b) and (d).

Ref. [23], where the ballooning stability diagrams for both the above ITER-like and highly-shaped plasmas have been identified.

In the following, the electrostatic ITG-mode stability and the residual zonal-flow levels are examined, where gyrokinetic ions and adiabatic electrons are considered. The fluxtube geometry on Boozer coordinates with $\rho=$ $\left(\Phi / \Phi_{\text {edge }}\right)^{1 / 2}$ is used, and we look into three different radial positions, i.e., $\rho=\{0.30,0.50,0.75\}$, which correspond to different flux-surface shapes. In order to focus on the impact of local shaping effects on the ITG-mode stability, the normalized density and temperature gradient parame- 
Table 1 Equilibrium parameters.

\begin{tabular}{l|ccccccccccc}
\hline & $R_{\mathrm{ax}}[\mathrm{m}]$ & $a[\mathrm{~m}]$ & $B_{\mathrm{ax}}[\mathrm{T}]$ & $I_{\mathrm{p}}[\mathrm{MA}]$ & $q_{95}$ & $\beta_{\mathrm{N}}$ & $\kappa_{95}^{\text {up }}$ & $\kappa_{95}^{\text {down }}$ & $\delta_{95}^{\text {up }}$ & $\delta_{95}^{\text {down }}$ & $S$ \\
\hline ITER-like & 3.10 & 1.32 & 2.51 & 2.49 & 4.32 & 2.02 & 1.63 & 1.83 & 0.29 & 0.44 & 3.25 \\
\hline highly-shaped & 3.18 & 1.61 & 2.61 & 5.00 & 3.97 & 3.11 & 1.82 & 1.81 & 0.46 & 0.43 & 4.72 \\
\hline
\end{tabular}
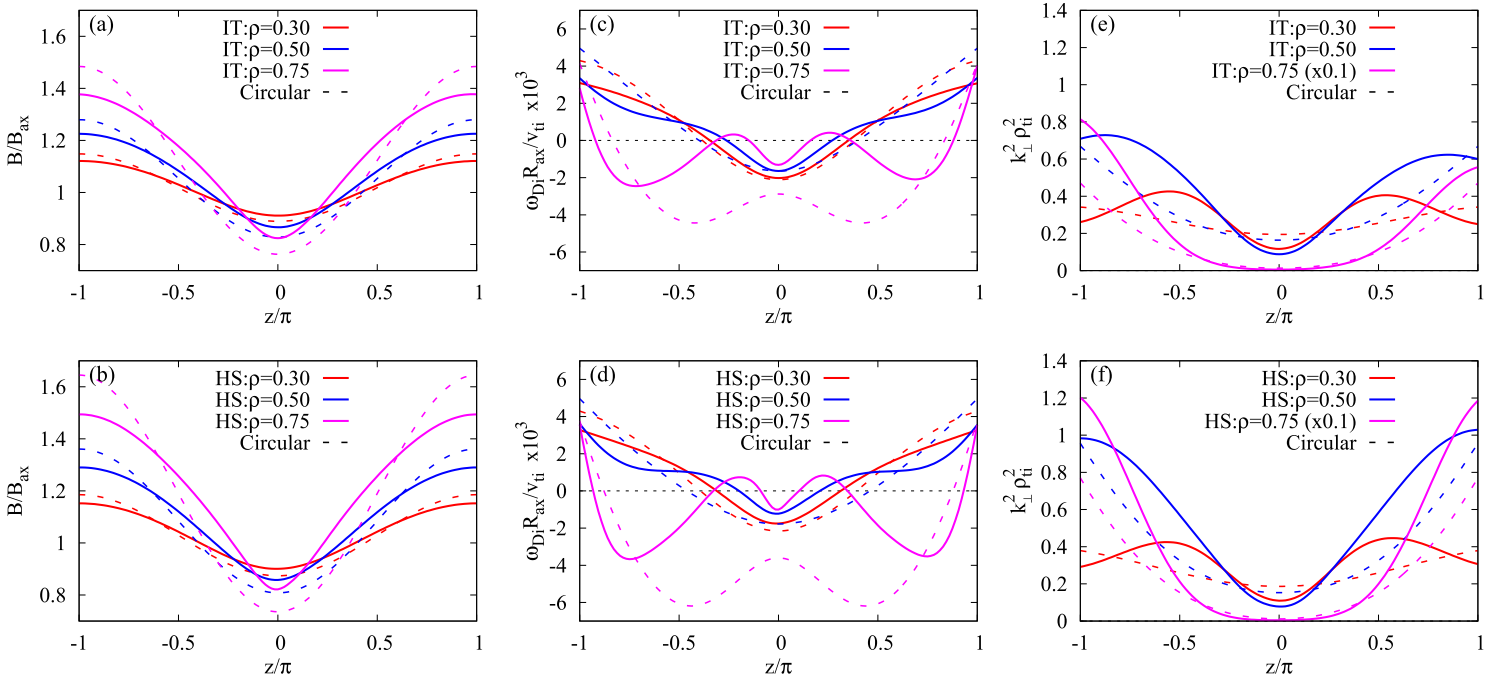

Fig. 6 Comparison of structures along the magnetic field lines at different $\rho$ for [(a),(b)]the magnetic field intensity $B$, [(c),(d)] the drift frequency $\omega_{\mathrm{Di}}\left(k_{x} \rho_{\mathrm{ti}}=0, k_{y} \rho_{\mathrm{ti}}=0.5, v_{\|} / v_{\mathrm{ti}}=2.5, \mu B_{\mathrm{ax}} / T_{\mathrm{i}}=0\right)$, and [(e),(f)]the squared perpendicular wavenumber $k_{\perp}^{2}\left(k_{x} \rho_{\mathrm{ti}}=0, k_{y} \rho_{\mathrm{ti}}=\right.$ $0.5)$, where the circular cases with same local values of $q, \hat{s}$ and $\epsilon$ are also plotted by dashed lines. IT: ITER-like, HS: highlyshaped.

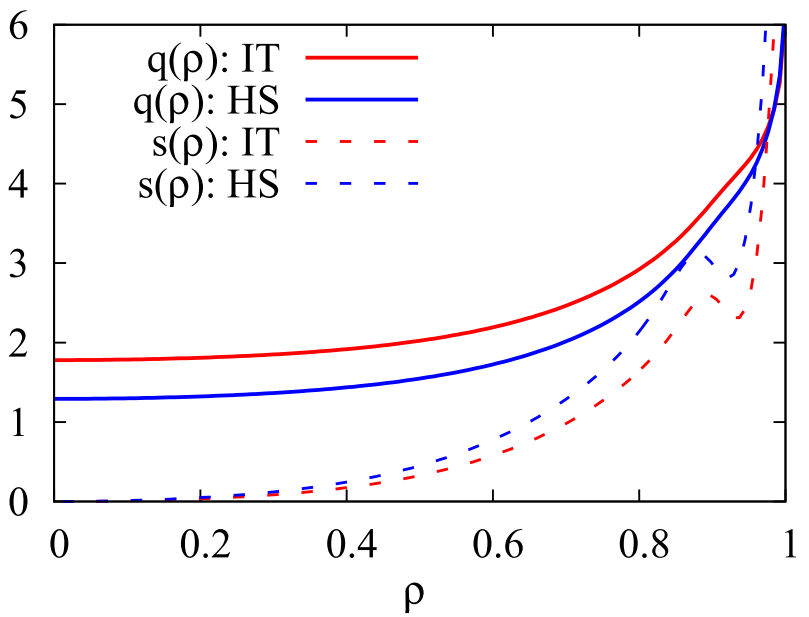

Fig. 5 Radial profiles of the safety factor $q$ and the magnetic shear $\hat{s}$ for the ITER-like (IT) and highly-shaped (HS) cases.

ters are assumed to be constant in the radial region of interest, where $R_{\mathrm{ax}} / L_{n_{\mathrm{i}}}=2.0$ and $R_{\mathrm{ax}} / L_{T_{\mathrm{i}}}=6.0$ with $T_{\mathrm{i}}=T_{\mathrm{e}}$. These are not the exact values obtained from equilibrium calculations, but are still feasible. Note also that these parameters appear only in $\omega_{* T_{\mathrm{i}}}$ [see Eq. (45)] which does not depend on geometrical quantities.

\subsection{Structures along confinement magnetic field}

Comparisons of the confinement field and linear advection operators, which are closely associated with the ITG-mode stability, are first presented in this section. In Fig. 6, the magnetic field intensity $B$, the drift frequency $\omega_{\text {Ds }}$, and the squared perpendicular wavenumber $k_{\perp}^{2}$ along the magnetic field line are compared between the ITERlike and highly-shaped plasmas, where the circular cases with same $q, \hat{s}$, and $\epsilon=\rho \epsilon_{a}$ shown in Appendix B are also plotted. One finds that the deviation of $B$ from the circular case becomes more significant for the outer side of the plasma [Figs.6(a) and 6(b)], where the magnetic field strength increases (decreases) in the low (high) field side of $z \sim 0(z \sim \pi)$. These features are also seen in the drift frequency $\omega_{\text {Ds }}$ for $\rho=0.30$ and $\rho=0.50$, while the qualitatively different structures with the multiple sets of nodes appear for $\rho=0.75$, and the deviation from the circular case is more pronounced for the highly-shaped case [Figs. 6(c) and 6(d)]. Similarly, in the squared perpendicular wavenumber $k_{\perp}^{2}$, the deviation becomes drastic for both the ITER-like and highly-shaped cases [Figs. 6 (d) and 6(e)], and then the slightly stronger up-down asymmetry is seen in the ITER-like case, e.g., the asymmetry at $\rho=0.5$ and $\rho=0.75$ is more apparent.

The comparison of the trapped-passing boundaries at 


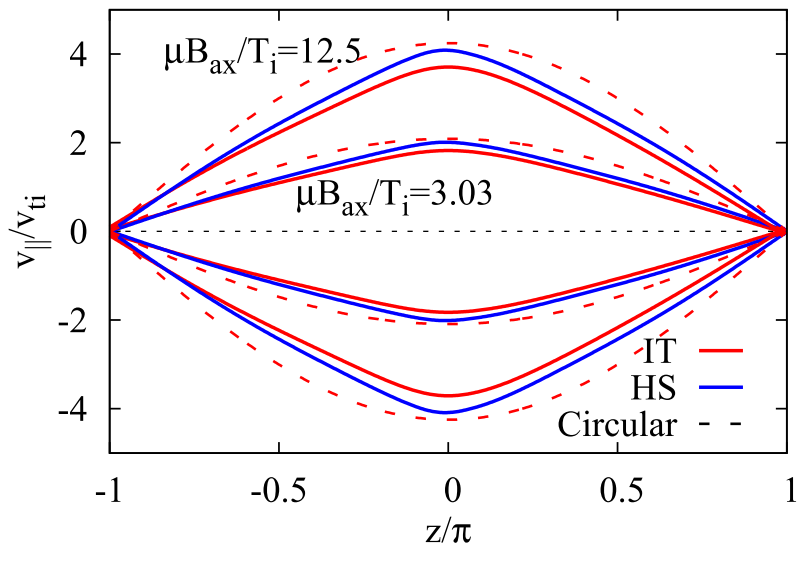

Fig. 7 Trapped-passing boundaries for different $\mu$-coordinates in (a) ITER-like and (b) highly-shaped equilibria, where the circular results are also plotted by dashed line. The highly-shaped case shows slightly wider region in comparison with the ITER-like case.

$\rho=0.75$ is shown in Fig. 7, where the circular cases are also plotted for the ITER-like plasma. As compared with the circular cases with the sinusoidal boundary shapes, the narrower trapped region with sharper boundaries is found. Also, the trapped region becomes slightly wider for the highly-shaped case, and these features indicate an impact on the trapped-electron mode (TEM) stability.

\subsection{Comparison of ITG-mode stability}

In this section, we make detailed comparisons on the ITG-mode stability between the ITER-like and highlyshaped equilibria. The linear growth rate $\gamma_{\mathrm{ITG}}$ and the real frequency $\omega_{\mathrm{r}}$ are shown in Fig. 8, where the corresponding circular cases are also plotted for comparisons. In the ITER-like case [Fig. 8 (a)], we see that, for $\rho=0.30$ and $\rho=0.50$, the maximum growth rate is similar to that in the circular case, while the profiles are shifted to the higher- $k_{y}$ region. The up-shift of $\gamma_{\mathrm{ITG}}$-profile is more significant for $\rho=0.75$, where the maximum growth rate also increases as compared to the circular case. The real frequency $\omega_{\mathrm{r}}$ also shows the up-shift feature with increasing $\rho$. The similar qualitative features on the spectral up-shift of $\gamma_{\mathrm{ITG}}$ and $\omega_{\mathrm{r}}$ are also identified for the highly-shaped case [Fig. 8 (b)], whereas the magnitude of the growth rate is lower than that in the ITER-like case for all three radial positions. Since the low-wavenumber modes around $k_{y} \rho_{\mathrm{ti}} \sim 0.5$ are, in general, considered to make dominant contributions to the ITG-driven turbulent transport, the stabilization in the lower- $k_{y}$ region are expected to reduce the transport levels.

A qualitative explanation on the physical mechanism leading to relatively lower growth rate in the highly-shaped case is given as follows. By considering a fluid limit ignoring the wave-particle resonance and finite gyroradius effects, the ITG-mode growth rate is evaluated by $\gamma_{\mathrm{ITG}} \simeq\left[\omega_{* P_{\mathrm{i}}} \omega_{\mathrm{D}_{\mathrm{i}}} /\left(1+\tau_{\mathrm{e}} k_{\perp}^{2} \rho_{\mathrm{ti}}^{2}\right)\right]^{1 / 2}$ for $\omega_{* P_{\mathrm{i}}} \omega_{\mathrm{D}_{\mathrm{i}}}>0$, where
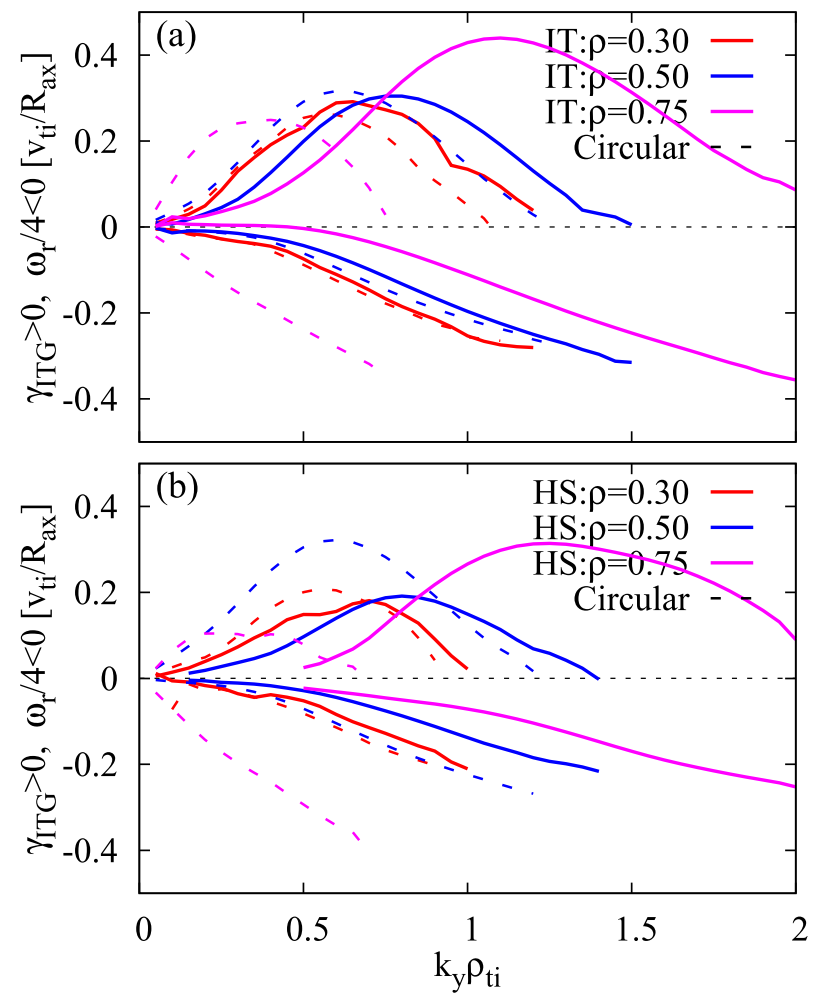

Fig. 8 Comparisons of linear ITG-mode dispersion relations among (a) ITER-like (IT) and (b) highly-shaped (HS) cases, where the circular results are shown by dashed lines. The highly-shaped case shows lower growth rate in comparison with the ITER-like case.

$\omega_{* P_{\mathrm{i}}}=-k_{y}\left(1+\eta_{\mathrm{i}}\right) c T_{\mathrm{i}} / e_{\mathrm{i}} c_{B} L_{n_{\mathrm{i}}} \leqslant 0, \tau_{\mathrm{e}}=T_{\mathrm{e}} / T_{\mathrm{i}}$, and $\omega_{\mathrm{D}_{\mathrm{i}}}(z)$ and $k_{\perp}^{2}(z)$ depend on the metric components [see. Eqs. (41) and (44)]. The reduction of $\left|\omega_{\mathrm{D}_{\mathrm{i}}}\right|$ and the increase of $k_{\perp}^{2}$ due to the shaping effect are, thus, responsible for the decrease of $\gamma_{\mathrm{ITG}}$. Actually, for $\omega_{\mathrm{D}_{\mathrm{i}}}<0$, the magnitude of $\left|\omega_{\mathrm{D}_{\mathrm{i}}}\right| /\left(1+\tau_{\mathrm{e}} k_{\perp}^{2} \rho_{\mathrm{ti}}^{2}\right)$ in the highly-shaped case is lower than that in the ITER-like one for all three radial positions.

In Fig. 9, linear eigenmode structures along the field lines are compared, where the eigenmodes giving the maximum growth rate are plotted. Reflecting the different structures of $B, \omega_{\mathrm{Ds}}$, and $k_{\perp}^{2}$ shown in Fig. 6, the deviation of the eigenmode profile from the circular case becomes larger as $\rho$ increases. One also finds that the highly-shaped case indicates more strongly localized peak near $\theta \sim 0$, as compared with the ITER-like (and also circular) case.

\subsection{Comparison of linear zonal-flow dynam- ics}

Finally, we present the comparisons of linear zonalflow evolutions in this section. Figure 10 shows time evolutions in the linear zonal-flow damping and the radialwavenumber $\left(k_{x} \rho_{\mathrm{ti}}\right)$ dependence of the residual zonal-flow levels for $\rho=0.30, \rho=0.50$, and $\rho=0.75$, where the circular cases are also plotted for the ITER-like cases. We see that the geodesic acoustic mode (GAM) frequency does 

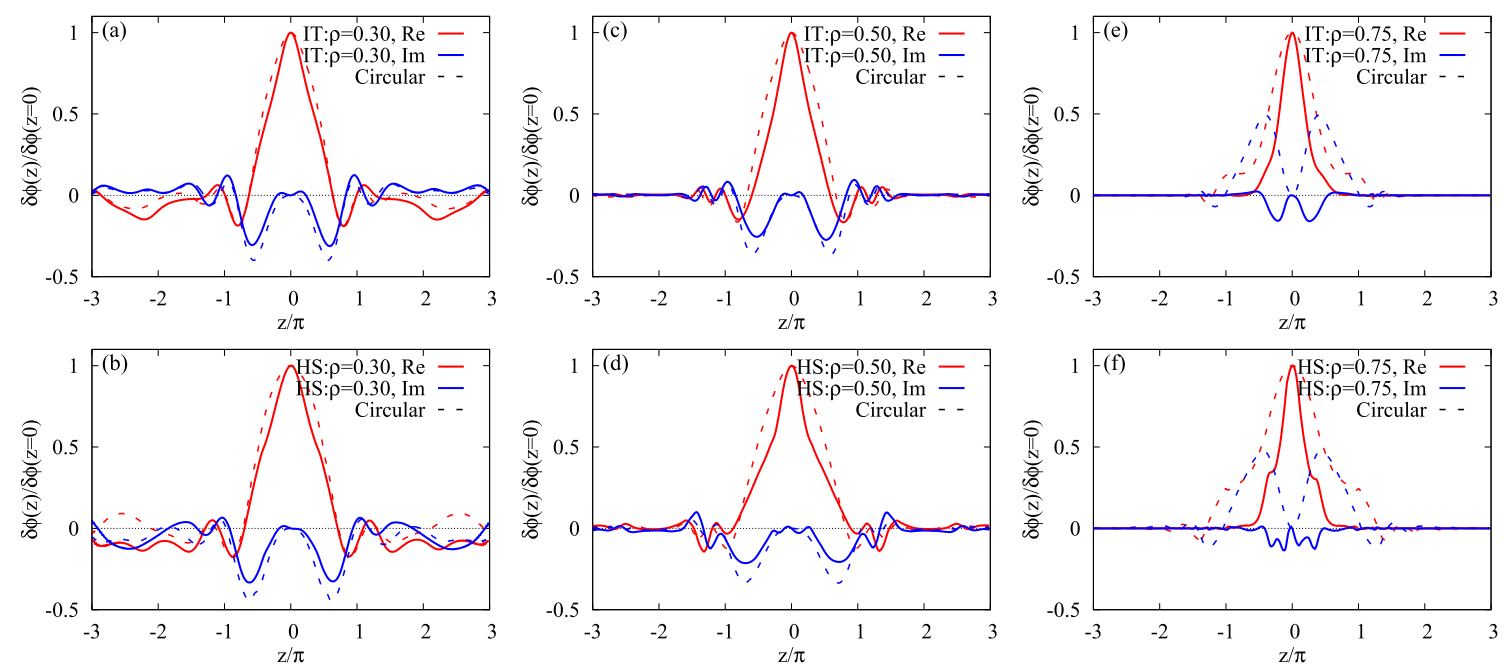

Fig. 9 Comparison of eigenmode structures giving the maximum growth rate for [(a), (c), (e)] ITER-like (IT) and [(b), (d), (f)] highlyshaped (HS) cases, where "Re" and "Im" represent the real and imaginary parts, respectively, and the circular cases are also shown by dashed lines.
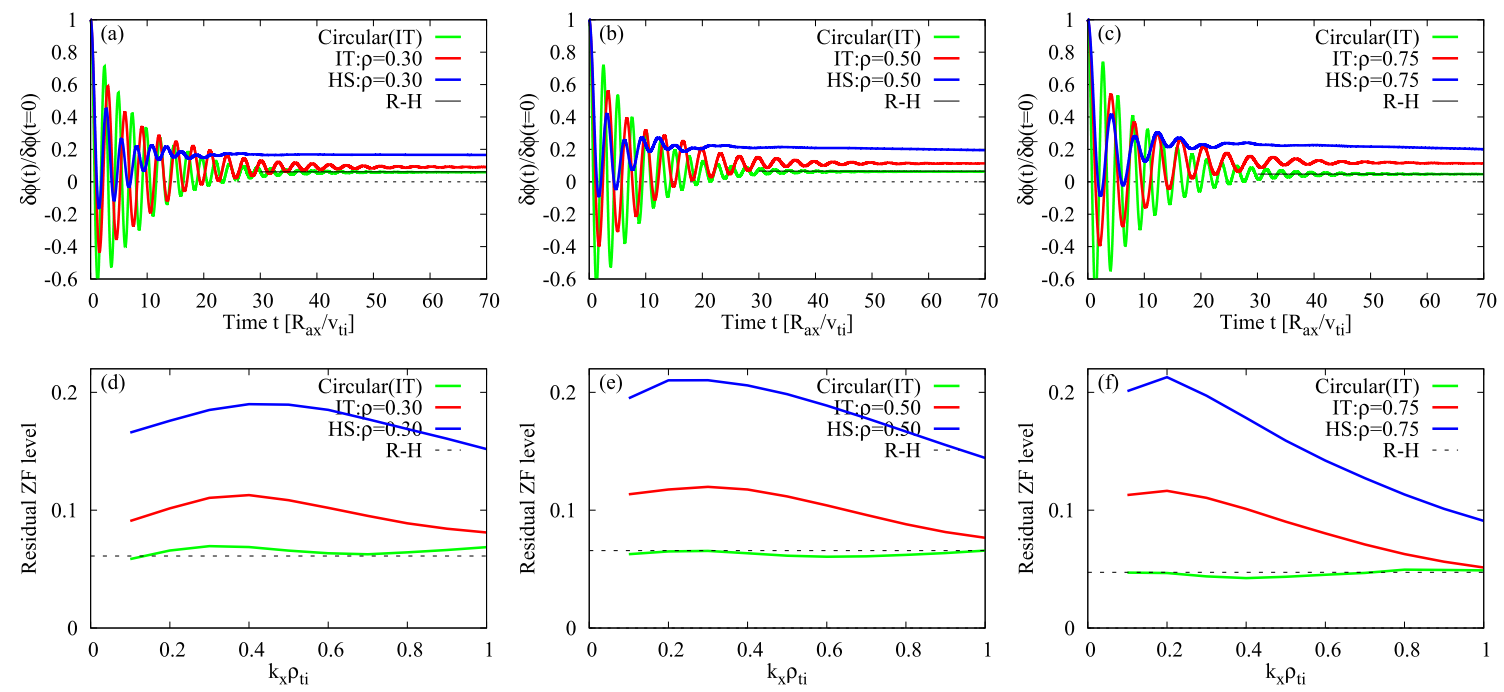

Fig. 10 [(a), (b), (c)] time evolutions in the linear zonal-flow damping for $k_{x} \rho_{\mathrm{ti}}=0.1$ at $\rho=0.30, \rho=0.50$, and $\rho=0.75$, and [(d), (e), (f)] the radial wavenumber dependencies of the residual zonal-flow level evaluated at $t=70$, where "R-H" shows the analytic circular result of $\left(1+1.6 q^{2} / \epsilon^{1 / 2}\right)^{-1}[15]$. The higher residual zonal-flow level is confirmed in the highly-shaped case for all three radial positions.

not change much in all the cases, while the residual zonalflow levels are enhanced as the shaping effects become more significant [Figs. 10 (a) - 10(c)], i.e., "circular case" $<$ "ITER-like case" < "highly-shaped case", and "inner side $(\rho=0.30)$ " < "mid-radius $(\rho=0.50)$ " < "outer side $(\rho=0.75)$ ". Particularly, for $k_{x} \rho_{\mathrm{ti}}=0.1$ which is a typical wavenumber for nonlinearly generated zonal flows in the ITG turbulence, approximately 2 times higher residual level is identified in the highly-shaped case.

Another important finding is difference in the radial wavenumber dependence of the residual level [Figs. 10 (d) - 10(f)]. As derived in Ref. [15], the $k_{x}$-dependence of the residual zonal-flow level in circular tokamaks appears only in the higher order in $\epsilon$. Indeed, such weak $k_{x}$-dependence is confirmed in the present numerical simulations with the circular equilibrium. In contrast, the strong $k_{x}$-dependence of the residual levels is revealed for both the ITER-like and highly-shaped cases, where almost linear dependencies with $-0.08 k_{x}$ for $\rho=0.30$ and $\rho=0.50$, and with $-0.16 k_{x}$ for $\rho=0.75$ are identified for $k_{x} \rho_{\mathrm{ti}}>0.5$.

\section{Summary}

Realistic tokamak geometries have been successfully implemented to a local fluxtube gyrokinetic Vlasov code GKV, by using a newly developed interface code 
IGS which can construct straight-field-line flux coordinates systems from MHD equilibria produced by a freeboundary 2D Grad-Shafranov equation solver MEUDAS. The accuracy of the flux coordinates, i.e., the axisymmetric, Boozer, and Hamada, constructed by IGS has been verified with the analytic Solov'ev equilibrium model. As another verification of GKV with IGS, cross-code benchmark test has been carried out by using Cyclone-base-case like MHD equilibrium, and good agreement in the dispersion relation of ITG-mode are confirmed.

By using the extended GKV, the ITG-mode stability and linear zonal-flow dynamics are investigated for two types of shaped plasmas expected in JT-60SA tokamak device, i.e., ITER-like and highly-shaped plasmas in which the MHD stability has been well examined. The detailed comparisons including the conventional circular equilibrium reveal that both the ITER-like and highly-shaped cases show the up-shift of $\gamma_{\mathrm{ITG}^{-}}$and $\omega_{\mathrm{r}}$-profiles to the higher- $k_{y}$ region in comparison with the circular cases. The maximum growth rate is similar to or less than the circular case for the inner sides of plasma $(\rho=0.30$ and $\rho=0.50)$, while the outer side with $\rho=0.75$ show the higher maximum growth rate at higher $k_{y}$. It is also found that, when the ITER-like and highly-shaped cases are compared, the latter case shows relatively lower growth rate for all the radial positions examined here, where the maximum growing eigenmode structure around $\theta \sim 0$ becomes sharper. Linear zonal-flow damping simulations clarify that the residual level is significantly enhanced by the shaping effects, and the radial wavenumber dependence becomes stronger in both the ITER-like and the highly-shaped cases.

From these analyses, it is expected that the highlyshaped equilibrium shows less ITG-driven turbulent transport than that in the ITER-like one, due to the reduction of the ITG-mode growth rate and the enhancement of residual zonal-flow levels. It should be noted that since the actual zonal-flow generation in ITG turbulence also depends on the turbulence intensity, which is well correlated to $\gamma_{\mathrm{ITG}} / k_{\perp}^{2}[35,36]$, the reduction of the ITG-mode growth rate can also decrease the source of zonal flows. However, the enhancement ratio of the residual zonal-flow level from the ITER-like to highly-shaped cases exceeds the reduction ratio of the ITG-mode growth rate. The detailed comparisons on the nonlinear zonal-flow generations and turbulent transport levels are demanded in a future work, and will be reported elsewhere.

\section{Acknowledgments}

The Authors would like to thank Dr. S. Brunner, Dr. J. Dominski, and Dr. X. Lapillonne for providing the CHEASE equilibrium data and benchmark results, and also thank Dr. Hideo Sugama, Dr. M. Honda, and Dr. M. Yoshida for fruitful discussions.

Numerical simulations were performed by HELIOS at Computational Simulation Centre of International Fu- sion Energy Research Centre, Oakleaf-FX at the University of Tokyo, and BX900 at Japan Atomic Energy Agency. This work is supported in part by the HPCI Strategic Program for Innovative Research, and in part by the NIFS Collaborative Research Program Nos. NIFS12KNTT014 and NIFS12KNTT015, and in part by the MEXT, Grant Nos. 22760660 and 23246163.

\section{Appendix A. Hamada, Boozer, and Axisymmetric Coordinates}

The fundamental properties of each coordinate system are briefly summarized here.

\section{A.1 Hamada coordinates ( $\tilde{\alpha}=0, f=$ Const.)}

$$
\begin{aligned}
\sqrt{g}_{H}(\rho) & =\frac{V^{\prime}}{4 \pi^{2}}, \\
B^{\theta_{H}}(\rho) & =\boldsymbol{B} \cdot \nabla \theta_{H}=4 \pi^{2} \frac{\Psi^{\prime}}{V^{\prime}}, \\
B^{\zeta_{H}}(\rho) & =\boldsymbol{B} \cdot \nabla \zeta_{H}=4 \pi^{2} \frac{\Phi^{\prime}}{V^{\prime}} .
\end{aligned}
$$

From Eqs. (1), (2), (4), (11), and (12), one finds

$$
\begin{aligned}
& \boldsymbol{B} \cdot \nabla \tilde{\gamma}_{H}=\left\langle B^{2}\right\rangle\left[\frac{B^{2}}{\left\langle B^{2}\right\rangle}-1\right], \\
& \boldsymbol{B} \cdot \nabla \tilde{\beta}_{H}=0 \Rightarrow \tilde{\beta}_{H}=\tilde{\beta}_{H}(\rho) .
\end{aligned}
$$

The equation (A.4) is the necessary and sufficient condition for Hamada coordinates, but one can always set $h^{\prime}(\rho)$ in Eq. (6) such that $\tilde{\beta}_{H}^{*}=0$, i.e., $h(\rho)=-\int_{0}^{\rho} \mathrm{d} \bar{\rho} \tilde{\beta}_{H}(\bar{\rho})$. Besides, no $h(\rho)$ exists to vanish $\tilde{\gamma}_{H}^{*}=\tilde{\gamma}_{H}\left(\rho, \theta_{H}, \zeta_{H}\right)-h(\rho)$ at any points in $\left(\theta_{H}, \zeta_{H}\right)$ for a given $\rho$. Then, the covariant components of $\boldsymbol{B}$ are given as

$$
\begin{aligned}
B_{\rho}\left(\rho, \theta_{H}, \zeta_{H}\right) & =\frac{\partial \tilde{\gamma}_{H}^{*}}{\partial \rho}, \\
B_{\theta_{H}}\left(\rho, \theta_{H}, \zeta_{H}\right) & =I+\frac{\partial \tilde{\gamma}_{H}^{*}}{\partial \theta_{H}}, \\
B_{\zeta_{H}}\left(\rho, \theta_{H}, \zeta_{H}\right) & =J+\frac{\partial \tilde{\gamma}_{H}^{*}}{\partial \zeta_{H}},
\end{aligned}
$$

where $\tilde{\gamma}_{H}^{*}$ is determined by Eq. (A.3) (Note that $\boldsymbol{B} \cdot \nabla \tilde{\gamma}_{H}=$ $\left.\boldsymbol{B} \cdot \nabla \tilde{\gamma}_{H}^{*}\right)$. Also, the contravariant components of $\boldsymbol{j}$ are given as

$$
j^{\theta_{H}}(\rho)=J^{\prime}, \quad j^{\zeta_{H}}(\rho)=I^{\prime}
$$

In Hamada coordinates, both the magnetic field and current density lines become straight on the flux surface. Although the Jacobian depending only on $\rho$ is significantly simplified (especially, for the flux-surface average), the covariant components of $\boldsymbol{B}$ become rather complicated.

\section{A.2 Boozer coordinates $\left(\tilde{\alpha}=0, f=B^{2}\right)$}

$$
\sqrt{g}_{B}\left(\rho, \theta_{B}, \zeta_{B}\right)=\frac{V^{\prime}}{4 \pi^{2}} \frac{\left\langle B^{2}\right\rangle}{B^{2}},
$$




$$
\begin{aligned}
& B^{\theta_{B}}\left(\rho, \theta_{B}, \zeta_{B}\right)=\boldsymbol{B} \cdot \nabla \theta_{B}=4 \pi^{2} \frac{\Psi^{\prime}}{V^{\prime}} \frac{B^{2}}{\left\langle B^{2}\right\rangle}, \\
& B^{\zeta_{B}}\left(\rho, \theta_{B}, \zeta_{B}\right)=\boldsymbol{B} \cdot \nabla \zeta_{B}=4 \pi^{2} \frac{\Phi^{\prime}}{V^{\prime}} \frac{B^{2}}{\left\langle B^{2}\right\rangle} .
\end{aligned}
$$

From Eqs. (1), (2), (4), (11), and (12), one finds

$$
\begin{aligned}
& \boldsymbol{B} \cdot \nabla \tilde{\beta}_{B}=p^{\prime}\left[1-\frac{B^{2}}{\left\langle B^{2}\right\rangle}\right], \\
& \boldsymbol{B} \cdot \nabla \tilde{\gamma}_{B}=0 \Rightarrow \tilde{\gamma}_{B}=\tilde{\gamma}_{B}(\rho) .
\end{aligned}
$$

The Eq. (A.12) is the necessary and sufficient condition for Boozer coordinates, but one can always set $h(\rho)$ in Eq. (7) such that $\tilde{\gamma}_{B}^{*}=0$. The covariant components of $\boldsymbol{B}$ are, then, given as

$$
\begin{aligned}
B_{\rho}\left(\rho, \theta_{B}, \zeta_{B}\right) & =\tilde{\beta}_{B}^{*}, \\
B_{\theta_{B}}(\rho) & =I, \\
B_{\zeta_{B}}(\rho) & =J,
\end{aligned}
$$

where $\tilde{\beta}_{H}^{*}$ is determined by Eq. (A.11) (Note that $\boldsymbol{B} \cdot \nabla \tilde{\beta}_{H}=$ $\left.\boldsymbol{B} \cdot \nabla \tilde{\beta}_{H}^{*}\right)$. The contravariant components of $\boldsymbol{j}$ are given as

$$
j^{\theta_{B}}\left(\rho, \theta_{B}, \zeta_{B}\right)=J^{\prime}+\frac{\partial \tilde{\beta}^{*}}{\partial \zeta_{B}}, \quad j^{\zeta_{B}}\left(\rho, \theta_{B}, \zeta_{B}\right)=I^{\prime}+\frac{\partial \tilde{\beta}^{*}}{\partial \theta_{B}} .
$$

In Boozer coordinates, only the magnetic field line becomes straight on the flux surface, but the covariant components of $\boldsymbol{B}$ are simplified. This feature is advantageous to solve numerically the guiding-center equations of motion.

\section{A.3 Axisymmetric coordinates $(\tilde{\alpha}=0, f=$} $\left.\boldsymbol{B} \cdot \nabla \zeta_{A}, \zeta_{A}=\phi\right)$

$$
\begin{aligned}
\sqrt{g}_{A}\left(\rho, \theta_{A}, \phi\right) & =\frac{V^{\prime}}{4 \pi^{2}}\left\langle R^{-2}\right\rangle R^{2}=\frac{\Phi^{\prime} R^{2}}{J}, \\
B^{\theta_{A}}\left(\rho, \theta_{A}, \phi\right) & =\boldsymbol{B} \cdot \nabla \theta_{A}=\frac{J}{q R^{2}}, \\
B^{\phi}\left(\rho, \theta_{A}, \phi\right) & =\boldsymbol{B} \cdot \nabla \phi=\frac{J}{R^{2}} .
\end{aligned}
$$

From Eqs. (1), (2), (4), (11), and (12), one finds

$$
\begin{aligned}
& \boldsymbol{B} \cdot \nabla \tilde{\beta}_{A}=p^{\prime}\left[1-\frac{R^{-2}}{\left\langle R^{-2}\right\rangle}\right], \\
& \boldsymbol{B} \cdot \nabla \tilde{\gamma}_{A}=\left\langle B^{2}\right\rangle\left[\frac{B^{2}}{\left\langle B^{2}\right\rangle}-\frac{R^{-2}}{\left\langle R^{-2}\right\rangle}\right] .
\end{aligned}
$$

In contrast to Hamada and Boozer ones, the axisymmetric coordinates show the finite $\boldsymbol{B} \cdot \nabla \tilde{\beta}_{A}$ and $\boldsymbol{B} \cdot \nabla \tilde{\gamma}_{A}$. It is, however, noted that when the poloidal magnetic field strength $B_{\text {pol }}=\Psi^{\prime}|\nabla \rho| R^{-1}$ is negligibly small, the axisymmetric coordinates coincide with Boozer one, i.e., $\boldsymbol{B} \cdot \nabla \tilde{\gamma}_{A} \rightarrow 0\left(B^{2} \rightarrow B_{\text {tor }}^{2}=J^{2} R^{-2}\right)$. The covariant components of $\boldsymbol{B}$ are given as

$$
B_{\rho}\left(\rho, \theta_{A}, \phi\right)=\tilde{\beta}_{A}+\frac{\partial \tilde{\gamma}_{A}}{\partial \rho}=-\frac{q \Psi^{\prime 2}}{J} \nabla \rho \cdot \nabla \theta_{A},(\mathrm{~A} .21)
$$

$$
\begin{aligned}
B_{\theta_{A}}\left(\rho, \theta_{A}, \phi\right) & =I+\frac{\partial \tilde{\gamma}_{A}}{\partial \theta_{A}}=\frac{q \Psi^{\prime 2}}{J} \nabla \rho \cdot \nabla \rho \\
B_{\phi}(\rho) & =J
\end{aligned}
$$

where, $\nabla \rho \cdot \nabla \phi=0, \nabla \theta_{A} \cdot \nabla \phi=0$, and $\partial / \partial \phi=0$ are used. Finally, the contravariant components of $\boldsymbol{j}$ are given as

$$
j^{\theta_{A}}(\rho)=J^{\prime}, \quad j^{\phi}\left(\rho, \theta_{A}, \phi\right)=I^{\prime}+\frac{\partial \tilde{\beta}}{\partial \theta_{A}} .
$$

In the axisymmetric coordinates, only $B_{\zeta}$ and $j^{\theta}$ are simplified, i.e., $B_{\zeta}=B_{\zeta}(\rho)$ and $j^{\theta}=j^{\theta}(\rho)$, and this coordinates are widely used for theoretical and numerical analyses of tokamak plasmas.

\section{Appendix B. Metric Components for Circular and $s-\alpha$ Models}

Analytic expressions of the concentric circular equilibrium and the simplified $s-\alpha$ one are summarized here. The detailed descriptions are shown in Ref. [34].

\section{B.1 Concentric circular equilibrium}

For toroidal plasmas with concentric circular flux surfaces, the axisymmetric coordinates $\left(\rho, \theta_{A}, \phi\right)$ are useful, where $\rho=\left(\Phi / \Phi_{\text {edge }}\right)^{1 / 2}=r / a$ with $a=\left(2 \Phi_{\text {edge }} / B_{\text {ax }}\right)^{1 / 2}$ is considered here. By using geometric polar-angle from the magnetic axis, $\theta_{0}$, in $\left(\rho, \theta_{0}, \phi\right)$, the SFL poloidal-angle coordinate $\theta_{A}$ is written by

$$
\theta_{A}=2 \tan ^{-1}\left[\left(\frac{1-\epsilon}{1+\epsilon}\right)^{\frac{1}{2}} \tan \left(\frac{\theta_{0}}{2}\right)\right]
$$

The magnetic flux surfaces for given $q$ are determined by $\mathrm{d} \Psi / \mathrm{d} r=B_{\mathrm{ax}} r / \bar{q}$, where $\bar{q}=\left(1-\epsilon^{2}\right)^{1 / 2} q$. Using these definitions, one can calculate the magnetic field intensity, its derivatives, and the contravariant metric components as follows: (Note that $q_{0}=q\left(\rho_{0}\right)$ and $\bar{q}_{0}=\bar{q}\left(\rho_{0}\right)$.)

$$
\begin{aligned}
B= & \left(1+\frac{\epsilon^{2}}{\bar{q}_{0}^{2}}\right)^{\frac{1}{2}} \frac{B_{\mathrm{ax}}}{1+\epsilon \cos \theta_{0}}, \\
\frac{\partial B}{\partial x}= & \frac{B}{B_{\mathrm{ax}} R_{\mathrm{ax}}\left(1+\epsilon \cos \theta_{0}\right)}\left[\cos \theta_{0}-\frac{\epsilon}{1-\epsilon^{2}} \sin ^{2} \theta_{0}\right. \\
& \left.-\frac{\epsilon\left(1-\hat{s}+\epsilon^{2} q_{0}^{2} / \bar{q}_{0}^{2}\right)}{\bar{q}_{0}^{2}+\epsilon^{2}}\right], \\
\frac{\partial B}{\partial z}= & \left(\frac{\bar{q}_{0}}{q_{0}}\right) \frac{B \epsilon \sin \theta_{0}}{1-\epsilon^{2} \cos \theta_{0} \cos \theta_{A}}, \\
\sqrt{g_{x y z}=} & q_{0} R_{\mathrm{ax}}\left(1+\cos \theta_{0}\right)^{2}, \\
g^{x x}= & \frac{q_{0}^{2}}{\bar{q}_{0}^{2}}, \quad g^{x y}=\frac{q_{0}^{2}}{\bar{q}_{0}^{2}}\left(\hat{s} \theta_{A}-\frac{\bar{q}_{0}}{q_{0}} \frac{\epsilon \sin \theta_{A}}{1-\epsilon^{2}}\right), \\
g^{x z}= & -\frac{q_{0}}{\bar{q}_{0}} \frac{\sin \theta_{a}}{1-\epsilon^{2}}, \\
g^{y y}= & \frac{q_{0}^{2}}{\bar{q}_{0}^{2}}\left[\hat{s}^{2} \theta_{A}^{2}-\frac{2 \bar{q}_{0}}{q_{0}} \hat{s} \theta_{A} \frac{\epsilon \sin \theta_{A}}{1-\epsilon^{2}}\right] \\
& +\left[\frac{\bar{q}_{0}^{2}}{q_{0}^{2}} \frac{\left(1+\epsilon^{2} / \bar{q}_{0}^{2}\right)}{\left(1+\epsilon \cos \theta_{0}\right)^{2}}+\frac{\epsilon^{2} \sin ^{2} \theta_{A}}{\left(1-\epsilon^{2}\right)^{2}}\right],
\end{aligned}
$$




$$
\begin{aligned}
& g^{y z}=\frac{1}{R_{\mathrm{ax}}}[-\frac{q_{0}}{\bar{q}_{0}} \hat{s} \theta_{A} \frac{\sin \theta_{A}}{1-\epsilon^{2}}+\frac{\bar{q}_{0}^{2}}{q_{0}^{2}} \frac{1}{\epsilon\left(1+\epsilon \cos \theta_{0}\right)^{2}} \\
&\left.+\frac{\epsilon^{2} \sin ^{2} \theta_{A}}{\left(1-\epsilon^{2}\right)^{2}}\right], \\
& g^{z z}=\frac{1}{R_{\mathrm{ax}}^{2}}\left[\frac{\bar{q}_{0}^{2}}{q_{0}^{2}} \frac{1}{\epsilon^{2}\left(1+\epsilon \cos \theta_{0}\right)^{2}}+\frac{\sin ^{2} \theta_{A}}{\left(1-\epsilon^{2}\right)^{2}}\right] .
\end{aligned}
$$

\section{B.2 $s-\alpha$ equilibrium model}

The so-called $s-\alpha$ equilibrium model used in gyrokinetic simulations is a simplified model of the above concentric circular one. By expanding Eqs. (B.1) - (B.10) with respect to $\epsilon$, but keeping the lowest or the first order terms, one can obtain the expressions in $s-\alpha$ model as follows: (Note that $q_{0}=\bar{q}_{0}$ and $\theta_{A}=\theta_{0}$.)

$$
\begin{aligned}
B & =B_{\mathrm{ax}}\left(1-\epsilon \cos \theta_{0}\right), \quad \frac{\partial B}{\partial x}=-\frac{B_{\mathrm{ax}} \cos \theta_{0}}{R_{\mathrm{ax}}}, \\
\frac{\partial B}{\partial z} & =B_{\mathrm{ax}} \epsilon \sin \theta_{0}, \quad \sqrt{g}_{x y z}=\frac{q_{0} R_{\mathrm{ax}} B_{\mathrm{ax}}}{B}, \\
g^{x x} & =1, \quad g^{x y}=\hat{s} \theta_{0}, \quad g^{x z}=0, \\
g^{y y} & =1+\hat{s}^{2} \theta_{0}^{2}, \quad g^{y z}=\frac{1}{R_{\mathrm{ax}} \epsilon}, \quad g^{z z}=\frac{1}{R_{\mathrm{ax}}^{2} \epsilon^{2}} .
\end{aligned}
$$

It should be noted that, in contrast to the concentric circular model, the $s-\alpha$ model no longer holds the consistency between the metric components and the magnetic field intensity given as $B^{2}=c_{B}^{2}\left[g^{x x} g^{y y}-\left(g^{x y}\right)^{2}\right]$.

[1] W. Horton, Rev. Mod. Phys. 71, 735 (1999).

[2] X. Garbet, Y. Idomura et al., Nucl. Fusion 50, 043002 (2010).

[3] A.M. Dimits, G. Bateman et al., Phys. Plasmas 7, 969 (2000).

[4] P.H. Diamond, S.-I. Itoh et al., Plasma Phys. Control. Fusion 47, R35 (2005).

[5] M.A. Beer, S.C. Cowley et al., Phys. Plasmas 2, 2687 (1995).

[6] R.L. Miller, M.S. Chu et al., Phys. Plasmas 5, 973 (1998).

[7] R.E. Waltz and R.L. Miller, Phys. Plasmas 6, 4265 (1999).

[8] E.A. Belli, G.W. Hammett et al., Phys. Plasmas 15, 092303 (2008).

[9] E.A. Belli and J. Candy, Phys. Plasmas 17, 112314 (2010).

[10] Y. Camenen, A. Pochelon et al., Nucl. Fusion 47, 510 (2007).

[11] A. Marinoni, S. Brunner et al., Plasma Phys. Control. Fusion 51, 055016 (2009).
[12] A. Burckel, O. Sauter et al., J. Phys. Conf. Ser. 260, 012006 (2010).

[13] T.L. Rhodes, C. Holland et al., Nucl. Fusion 51, 063022 (2011).

[14] R.V. Bravenec, J. Candy et al., Phys. Plasmas 18, 122505 (2011).

[15] M.N. Rosenbluth and F.L. Hinton, Phys. Rev. Lett. 80, 724 (1998).

[16] Y. Xiao and P.J. Catto, Phys. Plasmas 13, 082307 (2006).

[17] O. Yamagishi and H. Sugama, Phys. Plasmas 19, 092504 (2012).

[18] H. Sugama, T.-H. Watanabe et al., Phys. Plasmas 14, 022502 (2007)

[19] M. Nakata, T.-H. Watanabe et al., Phys. Plasmas 19, 022303 (2012).

[20] M. Nakata and Y. Idomura, Nucl. Fusion 53, 113039 (2013).

[21] T.-H. Watanabe and H. Sugama, Nucl. Fusion 46, 24 (2006).

[22] M. Azumi et al., in Proc. 4th Int. Symp. Comput. Methods Applied Sci. Engineering, Paris (North-Holland, Amsterdam, 1980) p.335.

[23] N. Aiba, S. Tokuda et al., Plasmas Fusion Res. 2, 010 (2007).

[24] N. Nakajima, J. Todoroki et al., Kakuyugo Kenkyu 68, 395 (1992).

[25] V.D. Pustovitov, J. Plasma Fusion Res. SERIES 1, 456 (1998).

[26] T. Takeda and S. Tokuda, J. Comput. Phys. 93, 1 (1991).

[27] M. Nunami, T.-H. Watanabe et al., Plasma Fusion Res. 5, 016 (2010).

[28] H. Sugama, T.-H. Watanabe et al., Phys. Plasmas 16, 112502 (2009).

[29] S. Maeyama, A. Ishizawa et al., Comput. Phys. Comm. 184, 2462 (2013).

[30] F. Jenko and W. Dorland, Plasma Phys. Control. Fusion 43, A141 (2001).

[31] W. Dorland, F. Jenko et al., Phys. Rev. Lett. 85, 5579 (2000).

[32] A.G. Peeters, Y. Camenen et al., Comput. Phys. Comm. 180, 2650 (2009).

[33] H. Lütjens, A. Bondeson et al., Comput. Phys. Comm. 97, 219 (1996).

[34] X. Lapillonne, S. Brunner et al., Phys. Plasmas 16, 032308 (2009).

[35] M. Nunami, T.-H. Watanabe et al., Phys. Plasmas 19, 042504 (2012).

[36] M. Nunami, T.-H. Watanabe et al., Phys. Plasmas 20, 092307 (2013) 